\title{
Interaction-induced harmonic frequency mixing in quantum dots
}

\author{
M. Thorwart, ${ }^{1}$ R. Egger, ${ }^{1}$ and A.O. Gogolin ${ }^{1,2}$ \\ 1 Institut für Theoretische Physik, Heinrich-Heine-Universität, D-40225 Düsseldorf, Germany \\ 2 Department of Mathematics, Imperial College, 180 Queen's Gate, London SW7 2BZ, UK
}

(Dated: November 8, 2018)

\begin{abstract}
We show that harmonic frequency mixing in quantum dots coupled to two leads under the influence of time-dependent voltages of different frequency is dominated by interaction effects. This offers a unique and direct spectroscopic tool to access correlations, and holds promise for efficient frequency mixing in nano-devices. Explicit results are provided for an Anderson dot and for a molecular level with phonon-mediated interactions.
\end{abstract}

PACS numbers: 73.23.-b, 72.10.-d, 73.50.Mx. 73.63.-b

The nonlinear mixing of two signals with different frequencies is a widespread and important concept used in many areas of physics. Frequency mixers have been extensively used for a long time in microelectronics [1], where a diode provides the required nonlinearity, and in nonlinear optics (three- and four-wave mixing) [2]. Submillimeter-wave heterodyne signal detection at the quantum noise limit [3], based on the nonlinear currentvoltage characteristics of a superconducting tunnel junction (STJ), is ubiquitous and has generated an enormous boost in radioastronomy. Apart from STJs, however, frequency mixing in quantum-coherent mesoscopic or nanodevices has been experimentally realized only for a threeterminal ballistic junction [4] and for the single-electron transistor [5, 6], the latter possessing a broad and tunable range of frequencies and bandwidths.

In this work, we give the quantum theory of frequency mixing in quantum dots - serving as prototype examples for nano-devices - in the presence of electron-electron (e-e) or electron-phonon (e-ph) interactions. Mesoscopic systems driven by ac fields are of major interest in condensed matter physics 7, 8, 9, 10, 11, 12, 13, 14, 15, 16, 17, 18], but a quantum theory of mixing has only been formulated for STJs [3]. We consider a multi-tone setup, where the dot is attached to source and drain contacts ("leads") with time-dependent (ac) voltages of different drive frequencies $\omega_{L / R}$ [19], and an additional gate is capacitively coupled to the dot. A quantity of main interest is the time-dependent current $I(t)$ through the device, expanded in a Fourier series as

$$
I(t)=\operatorname{Re} \sum_{n, m=-\infty}^{\infty} e^{-i \omega_{n m} t} I_{n m}
$$

with frequencies $\omega_{n m}=n \omega_{L}+m \omega_{R}$. By determining the complex-valued $I_{n m}$, we derive general conditions under which $I(t)$ exhibits harmonic frequency mixing. A mixing current can arise only if there exists at least one pair $(n>0, m \neq 0)$ with mixing amplitude $J_{n m} \equiv I_{n m}+I_{-n,-m}^{*} \neq 0$ (the star denotes complex conjugation). In order to establish the importance of interaction physics on frequency mixing, we explicitly compute the $J_{n m}$ for two important models, namely (i) for on-site e-e interaction $U$ in a spin-degenerate single-level dot (Anderson impurity model), and (ii) for a spinless level with e-ph coupling to an Einstein phonon, describing transport through vibrating molecules [20, 21].

Before turning to derivations, let us briefly summarize our main findings in simple qualitative terms. Taking the standard wide-band limit (WBL) for the leads, we find that no frequency mixing is possible in the absence of interactions. This is a striking and unexpected result that we shall discuss in some detail. Once interactions are present, however, one will generally encounter mixing, $J_{n m} \neq 0$ [22]. Therefore harmonic frequency mixing is dominated by interactions, and thus provides a highly sensitive novel spectroscopic tool of correlations. Importantly, we show that even in the linear response regime (dc bias voltage $V \rightarrow 0$ ) frequency mixing occurs in general, where the nonlinearity required for mixing is now generated by interactions. This implies that one may be able to operate such a quantum-coherent nano-device as frequency mixer with reduced dissipation and shot noise.

We here take the left/right $(\alpha=L / R= \pm)$ contact's single-particle energies as time-dependent, $\epsilon_{k, \alpha} \rightarrow$ $\epsilon_{k, \alpha} \pm e V / 2+V_{\alpha}^{a c} \cos \left(\omega_{\alpha} t\right)$, with dimensionless drive amplitudes $a_{\alpha}=V_{\alpha}^{a c} / \omega_{\alpha}$ (we put $\hbar=k_{B}=1$ ). The time-dependent current $I(t)=\left[I_{L}(t)-I_{R}(t)\right] / 2$ is then expressed in terms of $\Delta_{\alpha}(t)=a_{\alpha} \sin \left(\omega_{\alpha} t\right)$ and Fermi functions $f_{\alpha}(\omega)=f(\omega-\alpha e V / 2)$ describing the respective contact, and the exact but unknown retarded (lesser) Green's functions $(\mathrm{GF}) \mathbf{G}^{r}\left(t, t^{\prime}\right)\left(\mathbf{G}^{<}\left(t, t^{\prime}\right)\right)$ of the dot [9]. A time-dependent gate voltage then produces a similar term $\Delta_{g}(t)$. For simplicity, we assume that the tunneling amplitudes connecting the dot to the leads are not modulated by time-dependent voltages, and correspond to energy-independent hybridization matrices $\boldsymbol{\Gamma}_{\alpha}$ in the space of spin and dot level indices. By a gauge-invariant generalization of Ref. [9], the time-dependent currents $I_{\alpha}(t)$ for an interacting multi-level quantum dot can be written in the form

$$
\begin{aligned}
I_{\alpha}(t) & =-2 e \operatorname{Im} \operatorname{Tr}\left[\boldsymbol{\Gamma}_{\alpha} \mathbf{G}^{<}(t, t)\right] \\
& -2 e \operatorname{Im} \operatorname{Tr} \int d t^{\prime} \int \frac{d \omega}{2 \pi} f_{\alpha}(\omega) e^{i \omega\left(t-t^{\prime}\right)} \\
& \times e^{-i\left[\delta_{\alpha}(t)-\delta_{\alpha}\left(t^{\prime}\right)\right]} \boldsymbol{\Gamma}_{\alpha} \mathbf{G}^{r}\left(t, t^{\prime}\right),
\end{aligned}
$$

where $\delta_{\alpha}(t)=\Delta_{\alpha}(t)-\Delta_{g}(t)$. 
The formula (2) is manifestly gauge-invariant: adding an arbitrary time-dependent voltage to all (source, drain, and gate) electrodes leaves $I_{\alpha}(t)$ unaffected. However, Eq. (2) per se does not include displacement currents [23], which are generally necessary for conservation of total charge in time-dependent quantum transport [11, 16, 17, 18]. Moreover, they generate a drivinginduced energy renormalization of the dot states [11, 16], which has to be determined self-consistently. On general grounds, Fransson [16] has shown that for arbitrary drive frequencies, the self-consistently evaluated displacement currents can be separated into independent (left and right) parts. Thus, they cannot contribute to the mixing amplitudes (1), and we shall disregard them from now on. Having established gauge invariance, we also put $\Delta_{g}(t)=0$ in what follows.

Using Eq. (2), it can now be shown that in the absence of interactions, frequency mixing is suppressed by the parametrically small ratio $\operatorname{Tr}\left(\boldsymbol{\Gamma}_{L / R}\right) / D$, where $D$ is the electronic bandwidth in the leads. Therefore, in the wide band limit for the leads, no frequency mixing occurs for a noninteracting quantum dot, $J_{n m}=0$, regardless of how complicated its level structure may be. Technically, this follows because in the WBL the noninteracting GF $\mathbf{G}_{0}^{r}\left(t, t^{\prime}\right)$ is independent of the ac drive, while $\mathbf{G}_{0}^{<}(t, t)$ depends on the left/right drive in an additive way only [9]. One therefore encounters only Fourier components $I_{n m} \neq 0$ for $n=0$ or $m=0$ in Eq. (1). The WBL for the leads is known to provide an excellent description when transport is dominated by states close to the Fermi level where $\boldsymbol{\Gamma}_{\alpha}$ has only weak energy dependence. Note that the same conclusion holds true for $\left[I_{L}(t)+I_{R}(t)\right] / 2$, as the "no mixing theorem" applies to $I_{L}$ and $I_{R}$ separately. Below we focus on the $J_{n m}$ extracted from $I(t)=\left[I_{L}(t)-\right.$ $\left.I_{R}(t)\right] / 2$, and adopt the WBL, where $J_{n m} \neq 0$ can only be caused by interactions. Moreover, from now on we assume that only a single dot level is relevant and take symmetric hybridization $\Gamma_{L}=\Gamma_{R}=\Gamma / 2$. The respective generalizations are straightforward but do not yield new physics. The absence of mixing in the noninteracting limit may come as a surprise, since even in the WBL the $I V$ curve of an undriven single-level dot is nonlinear. For instance, for a noninteracting resonant level at $T=0$, one finds $I(V)=\frac{2 e \Gamma}{h} \tan ^{-1}(e V / 2 \Gamma)$. While this nonlinearity allows to generate higher harmonics and rectification [14, 18], it does not create finite mixing amplitudes in Eq. (11). The latter arise from an effective "cross-talk" between the source and drain electrodes, which can only be mediated by interactions.

The fact that interactions induce mixing can be demonstrated on the simplest possible level by firstorder perturbation theory in $U$ for the Anderson dot. The retarded self energy is then given by $\Sigma^{r}\left(t, t^{\prime}\right)=$ $U n(t) \delta\left(t-t^{\prime}\right)$, with the time-dependent dot occupation $n(t)=-i G_{0}^{<}(t, t)$. Expanding in Bessel functions, a closed expression for $n(t)$ in terms of the free GF and Fermi functions follows. Notably, in the driven case, the dot occupation and hence $\Sigma^{r}$ become time-dependent, while for the time-independent case, there is only a rigid shift of the dot level. The GF correction $\Delta G^{r}=G_{0}^{r} \Sigma^{r} G_{0}^{r}$ now generates mixing, and after some algebra, we obtain the mixing coefficients to first order in $U(n, m \neq 0)$,

$$
\begin{aligned}
I_{n m} & =i U \sum_{k l=-\infty}^{\infty} J_{k+n}\left(a_{L}\right) J_{k}\left(a_{L}\right) J_{l+m}\left(a_{R}\right) J_{l}\left(a_{R}\right) \\
& \times\left[F_{a}^{R}\left((l+m) \omega_{R}, l \omega_{R}\right) F_{r}^{L}\left(-k \omega_{L},-k \omega_{L}-m \omega_{R}\right)\right. \\
& \left.-F_{a}^{L}\left((k+n) \omega_{L}, k \omega_{L}\right) F_{r}^{R}\left(-l \omega_{R},-l \omega_{R}-n \omega_{L}\right)\right],
\end{aligned}
$$

with the auxiliary functions

$$
F_{r / a}^{R / L}\left(\omega_{1}, \omega_{2}\right)=\Gamma \int \frac{d \epsilon}{2 \pi} f_{R / L}(\epsilon) G_{0}^{r}\left(\epsilon+\omega_{1}\right) G_{0}^{r / a}\left(\epsilon+\omega_{2}\right),
$$

which can be evaluated in closed (but lengthy) form. They obey the symmetry relations $F_{r}\left(\omega_{1}, \omega_{2}\right)=$ $F_{r}\left(\omega_{2}, \omega_{1}\right)$ and $F_{a}\left(\omega_{1}, \omega_{2}\right)=F_{a}^{*}\left(\omega_{2}, \omega_{1}\right)$. Elementary inspection of Eq. (3) shows that for $V=0, I_{n m} \rightarrow-I_{m n}$ for $\omega_{R} \rightarrow \omega_{L}$, as expected when exchanging source/drain contacts. Eq. (3) nicely illustrates the basic mechanism: for $U \neq 0$, terms containing $\omega_{L}$ and $\omega_{R}$ appear in a multiplicative way, showing that mixing is due to "cross-talk" of the leads. For zero dc bias $(V=0)$ and $\epsilon_{0}=0$, Eq. (3) and the above symmetry relations for $F_{r / a}$ imply $J_{n m}=0$ for all even $n+m$. Frequency mixing in this particle-hole symmetric limit thus disappears, say, at the difference frequency $\omega_{1,-1}=\omega_{L}-\omega_{R}$, but is still present at $\omega_{2,-1}$. However, once $V \neq 0$ or $\epsilon_{0} \neq 0$, one always finds mixing. To see this, it is instructive to consider very small drive amplitudes $V_{L, R}^{a c}$ in Eq. (3). Upon expanding the Bessel functions, the mixing amplitudes $J_{n m} \propto a_{L} a_{R}$ are nonzero only for $n, m= \pm 1$, and the double sum (3) receives contributions only from $k=0,-n$ and $l=0,-m$. Since $n+m$ is now always even, there is no mixing unless $V \neq 0$ or $\epsilon_{0} \neq 0$. This simple calculation already demonstrates that mixing is generated by interactions.

To study interaction effects beyond lowest order, we next consider the sequential tunneling regime with $T>$ $\Gamma$, where a master equation approach applies. Here one evaluates the dynamics of the occupation probabilities $P_{s}(t)$ [with $0 \leq P_{s}(t) \leq 1$ and $\sum_{s=1}^{4} P_{s}(t)=1$ ] for the four possible dot configurations $s$ with energy $\epsilon_{s}: s=1$ denotes the empty $\operatorname{dot}\left(\epsilon_{1}=0\right), s=2,3$ the singlyoccupied dot with spin up/down $\left(\epsilon_{2,3}=\epsilon_{0}\right)$, and $s=$ 4 the doubly occupied one $\left(\epsilon_{4}=2 \epsilon_{0}+U\right)$. Following standard steps [10], the master equation reads $\dot{P}(t)=$ $\sum_{s^{\prime}, \alpha=L / R}\left[K_{s s^{\prime}}^{\alpha}(t) P_{s^{\prime}}(t)-K_{s^{\prime} s}^{\alpha}(t) P_{s}(t)\right]$ with transition rates $\left(s \neq s^{\prime}\right.$, where $\left.K_{s s}^{\alpha}=-\sum_{s^{\prime} \neq s} K_{s s^{\prime}}^{\alpha}\right)$

$$
\begin{aligned}
K_{s s^{\prime}}^{\alpha}(t) & =\Gamma_{\alpha} \operatorname{Re} \sum_{k, q=-\infty}^{\infty} e^{i q \omega_{\alpha} t} J_{k}\left(a_{\alpha}\right) J_{k+q}\left(a_{\alpha}\right) \\
& \times \sum_{ \pm} N_{ \pm} g_{ \pm}\left( \pm\left[\epsilon_{s}-\epsilon_{s^{\prime}}\right]+k \omega_{\alpha}-\alpha e V / 2\right),
\end{aligned}
$$

where $N_{+}=\sum_{\sigma=\uparrow, \downarrow}\left|\left\langle s\left|d_{\sigma}^{\dagger}\right| s^{\prime}\right\rangle\right|^{2}, N_{-}$follows by replacing 
(a)

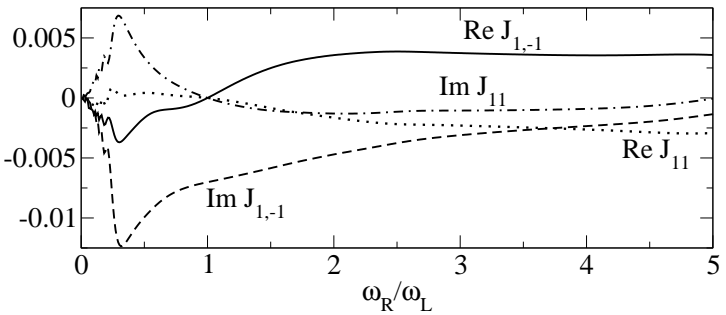

(b)

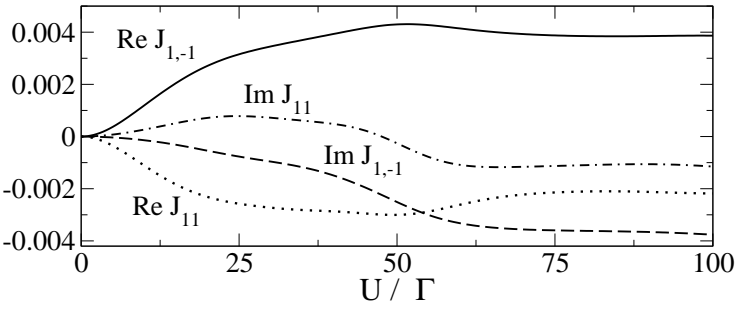

FIG. 1: Mixing amplitudes $J_{1, \pm 1}$ in the sequential tunneling regime, in units of $2 e \Gamma / \hbar$ for $V=\epsilon_{0}=0, \omega_{L}=20 \Gamma, V_{L}^{a c}=$ $V_{R}^{a c}=50 \Gamma$ and $T=5 \Gamma$, as a function of (a) $\omega_{R} / \omega_{L}$ for $U=100 \Gamma$, and (b) of $U / \Gamma$ for $\omega_{R}=50 \Gamma$.

the dot fermion operator $d_{\sigma}^{\dagger} \rightarrow d_{\sigma}$, and

$$
g_{ \pm}(\epsilon)=f(\epsilon) \pm \frac{i}{\pi} \ln \frac{D}{2 \pi T} \mp \frac{i}{\pi} \operatorname{Re} \psi\left(\frac{1}{2}+\frac{i \epsilon}{2 \pi T}\right),
$$

where $\psi$ is the digamma function, implying logarithmic divergencies [24]. After Fourier expansion as in Eq. (1), the master equation leads to an algebraic equation. Numerical solution obtains the Fourier coefficients $P_{s, n m}$ and hence the $J_{n m}$ from $I_{\alpha}(t)=\sum_{s \neq s^{\prime}} \theta_{s s^{\prime}} K_{s s^{\prime}}^{\alpha}(t) P_{s^{\prime}}(t)$, where $\theta_{s s^{\prime}}= \pm 1$ for $s \gtrless s^{\prime}$. These equations allow to reproduce known results for the sequential tunneling current under a dc bias and for the ac driven case with $\omega_{R}=\omega_{L}$ [10]. For $U=0$, in accordance with our discussion above, no frequency mixing is found from the master equation, while $J_{n m} \neq 0$ for $U \neq 0$. To ensure consistency, we have checked that for small $U$, the master equation reproduces the perturbative result (3) taken at high $T$ or large $V$.

Figure 1 shows the mixing amplitudes $J_{1, \pm 1}$ for $V=$ $\epsilon_{0}=0$ as a function of $U / \Gamma$ and $\omega_{R} / \omega_{L}$. Note that for $\omega_{R}=\omega_{L}$, we correctly find $J_{11}=0$ as enforced by the $V=0$ symmetry $I_{n m}=-I_{m n}$ under exchange of $\omega_{R}$ and $\omega_{L}$. At this point, $\operatorname{Re} J_{1,-1}$ also vanishes, while $\operatorname{Im} J_{1,-1} \neq$ 0 does not generate current, $I(t) \propto \sin \left[\left(\omega_{R}-\omega_{L}\right) t\right]=0$. Remarkably, the mixing amplitudes display characteristic features (peaks or steps) at certain ratios $\omega_{R} / \omega_{L}$, cp. for $\omega_{R} / \omega_{L}=1 / 3$ in Fig. 1(a). Such features can be rationalized in simple terms by noting that the ac voltages correspond to photon-assisted side peaks in the dot's density of states, located at energies $\epsilon_{0} \pm \omega_{n m}$ and $2 \epsilon_{0}+U \pm \omega_{n m}$ with arbitrary integers $n, m$. Once one of those energies hits the Fermi level (which is located at $\epsilon_{0}=0$ in Fig. 1), transition amplitudes are resonantly enhanced, and mixing becomes particularly efficient. To give an example, such resonances occur for $\omega_{R} / \omega_{L}=\left(U / \omega_{L}-n\right) / m$, and in Fig. 1(a), where $U / \omega_{L}=5$, the feature at $\omega_{R} / \omega_{L}=1 / 3$ corresponds to $(n, m)=(4,3)$. Which of these commensurability features (indexed by $n, m$ ) will actually show up in the mixing current is primarily determined by the drive amplitudes $V_{L / R}^{a c}$. For the rather large $V_{L / R}^{a c}$ taken in Fig. 1, large $(n, m)$ have to be taken into account, resulting in the rather complicated dependence of the mixing amplitudes on $\omega_{R} / \omega_{L}$ observed for $\omega_{R} / \omega_{L}<1 / 3$. The data in Fig. 1(a) show that mixing disappears in the limit $\omega_{L} \rightarrow \infty$. We also observe that mixing disappears in the opposite limit $\omega_{R} / \omega_{L} \rightarrow \infty$, where one can effectively average over the fast $\omega_{R}$ oscillations and ends up with a monochromatic situation again. Finally, additional calculations (not shown) reveal that like in the small- $U$ case, there is no mixing $\left(J_{n m}=0\right)$ at the particle-hole symmetric point, $\epsilon_{0}=-U / 2$ and $V=0$, for all even $n+m$. Before turning to the case of e-ph interactions, we briefly comment on the Kondo regime, realized at low temperatures for $U \gg \Gamma$ and $\epsilon_{0} \approx-U / 2$, where one can map the Anderson dot to a spin- $1 / 2$ impurity problem. At the special Toulouse point, an exact solution of the mixing problem can then be obtained from Ref. [13]. This solution will be discussed elsewhere, but by taking $T=V=0$, mixing is seen to disappear, in accordance with the Fermi liquid nature of the Kondo fixed point. At finite $T, V$, however, we again find nonzero mixing amplitudes.

Next we discuss a spinless level (fermion operator $d$ ), with e-ph coupling $\lambda$ to a phonon mode $Q=b+b^{\dagger}$ of frequency $\Omega$ (boson operator $b$ ). The dot is described by $H_{\mathrm{dot}}=\left[\epsilon_{0}+\lambda Q\right] d^{\dagger} d+\Omega b^{\dagger} b$, and connected to leads as for the Anderson dot. We have computed the mixing amplitudes $J_{n m}=I_{n m}+I_{-n,-m}^{*}$ by second-order perturbation theory in $\lambda$, with the result $I_{n m}=\left[I_{n m}^{+}-I_{m n}^{-}\right] / 2$, where

$$
\begin{aligned}
I_{n m}^{\alpha} & =i(\lambda \Gamma / 2 \pi)^{2} \sum_{k, l} J_{l+n}\left(a_{\alpha}\right) J_{l}\left(a_{\alpha}\right) J_{k+m}\left(a_{-\alpha}\right) J_{k}\left(a_{-\alpha}\right) \\
& \times \int d \omega \int d \epsilon f_{\alpha}(\omega) f_{-\alpha}(\epsilon) D_{0}^{r}\left(\omega-\epsilon-l \omega_{\alpha}-k \omega_{-\alpha}\right) \\
& \times G_{0}^{r}\left(\epsilon+(k+m) \omega_{-\alpha}\right) G_{0}^{a}\left(\epsilon+k \omega_{-\alpha}\right) G_{0}^{r}\left(\omega-l \omega_{\alpha}\right) \\
& \times G_{0}^{r}\left(\omega-l \omega_{\alpha}+m \omega_{-\alpha}\right),
\end{aligned}
$$

where $D_{0}^{r}(\omega)=2 \omega /\left[\left(\omega+i 0^{+}\right)^{2}-\Omega^{2}\right]$. From these expressions, one shows that for $V=\epsilon_{0}=0$, again $J_{n m} \neq 0$ only for even $n+m$. For $\Omega \gg \Gamma$, the high phonon frequency allows to approximate the boson propagator by a constant, and one essentially comes back to the Anderson dot result (3) where mixing was established above. In Fig. 2, we show the mixing amplitudes for $\Omega=\Gamma$ and small drive amplitudes in Eq. (6).

Finally, we comment on how frequency mixing could be verified experimentally, and on consequences for applications. Working quantum-dot frequency mixers [5, 6] operate in the adiabatic regime at rather low $\omega_{R / L}$ (no photon-assisted effects), probably outside the applicability range of our theory [19]. The measured dot capacitance $C=550 \mathrm{aF}$ [5] puts $U=e^{2} / 2 C$ around $35 \mathrm{GHz}$, while $\Gamma=2 \pi / R C \approx 13 \mathrm{GHz}$ follows from the measured 


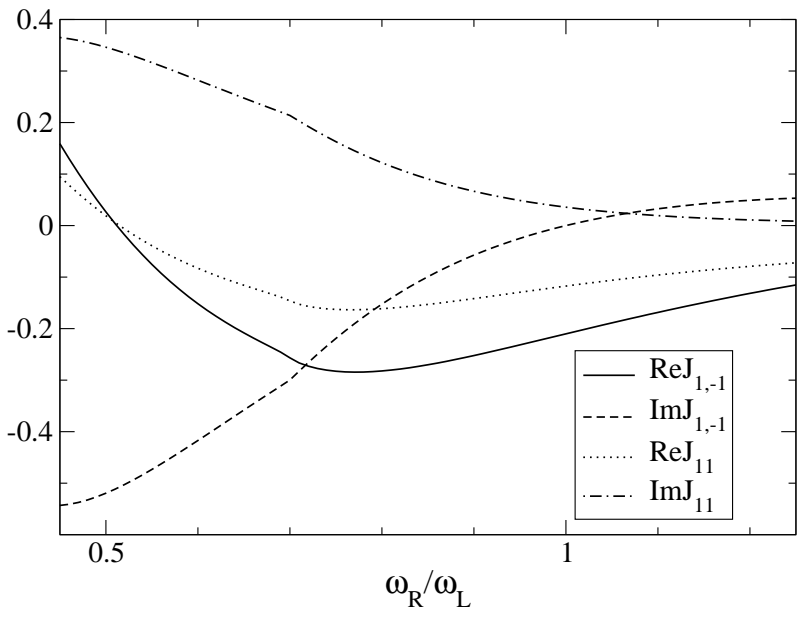

FIG. 2: Mixing amplitudes $J_{n m}$ for phonon-mediated interactions and small drive amplitudes $V_{L / R}^{a c}$, see Eq. (6) expanded to order $V_{L}^{a c} V_{R}^{a c}$. Parameters: $T=\epsilon_{0}=0, \Omega=$ $\Gamma, V=0.8 \Gamma, \omega_{L}=2 \Gamma$. The $J_{n m}$ are given in units of $\lambda^{2} V_{L}^{a c} V_{R}^{a c} /(2 \pi \Gamma)^{3}$.

resistance $R=850 \mathrm{k} \Omega$, implying $U / \Gamma \approx 2.7$. By comparing to Fig. 1] a large mixing signal is expected for $U / \Gamma \approx 50$, which may be achieved for the same $C$ by increasing $R$ to $R \approx 23 \mathrm{M} \Omega$, leading to $\Gamma=500 \mathrm{MHz}$. Choosing $\omega_{R / L}$ in the $\mathrm{GHz}$ range, see Fig. 1, should then put the device into an experimentally accessible regime, where our theory applies and predicts strong frequency mixing. In fact, one may be able to generate $\mathrm{THz}$ waves by such a mixer. In the present work, we have shown that mixing is possible for $V=0$, where the dc current vanishes and hence a very small noise level is expected. Existing experiments [5] involved finite dc current, causing large noise levels and dissipation effects in the detection electronics. Both these problems can be considerably reduced by using a correlated dot near $V=0$, where interactions provide the required nonlinearity.

To conclude, the theory of harmonic frequency mixing in interacting quantum dots has been given. In the wide-band limit for the leads, a "no mixing theorem" can be established, stating that mixing requires the presence of interactions. For both e-e and e-ph interactions, we have then shown that mixing is indeed generated, and provided detailed quantitative predictions for the mixing amplitudes. We hope that these findings stimulate experiments and further theoretical developments.

We thank M. Devoret for inspiring this work, and K. Flensberg, A.P. Jauho, Yu. Nazarov, J. Paaske, M. Polianski, and H. Schoeller for discussions. This work was supported by the DFG SFB TR 12 and by the ESF network INSTANS. A.O.G. thanks the Humboldt foundation for a Friedrich-Wilhelm-Bessel award enabling his extended stay in Düsseldorf.
[1] D.M. Pozar, Microwave Engineering, 2nd ed. (Wiley, New York, 1998).

[2] S. Mukamel, Principles of nonlinear optical spectroscopy (Oxford University Press, 1995).

[3] J.R. Tucker and M.J. Feldman, Rev. Mod. Phys. 57, 1055 (1985); P. Kittara, S. Withington, and G. Yassin, J. Appl. Phys. 101, 024508 (2007).

[4] J. Sun, D. Wallin, P. Brusheim, I. Maximov, Z.G. Wang, and H.Q. Xu, Nanotechnology 18, 195205 (2007).

[5] R. Knobel, C.S. Yung, and A.N. Cleland, Appl. Phys. Lett. 81, 532 (2002); L.J. Swenson, D.R. Schmidt, J.S. Aldridge, D.K. Wood, and A.N. Cleland, Appl. Phys. Lett. 86, 173112 (2005).

[6] D.J. Reilly and T.M. Buehler, Appl. Phys. Lett. 87, 163122 (2005).

[7] G. Platero and R. Aguado, Phys. Rep. 395, 1 (2004).

[8] S. Kohler, J. Lehmann, and P. Hänggi, Phys. Rep. 406, 379 (2004).

[9] A.P. Jauho, N.S. Wingreen, and Y. Meir, Phys. Rev. B 50, 5528 (1994).

[10] C. Bruder and H. Schoeller, Phys. Rev. Lett. 72, 1076 (1994).

[11] M.H. Pedersen and M. Büttiker, Phys. Rev. B 58, 12993 (1998).

[12] M. Hettler and H. Schoeller, Phys. Rev. Lett. 74, 4907 (1995); A. Kaminski, Yu.V. Nazarov, and L.I. Glazman, Phys. Rev. B 62, 8154 (2000); Y. Goldin and Y. Avishai, Phys. Rev. B 61, 16750 (2000); R. López, R. Aguado, G. Platero, and C. Tejedor, Phys. Rev. B 64, 075319 (2001).
[13] A. Schiller and S. Hershfield, Phys. Rev. Lett. 77, 1821 (1996).

[14] Y. Goldin and Y. Avishai, Phys. Rev. B 55, 16359 (1997).

[15] B. Wang, J. Wang, and H. Guo, Phys. Rev. Lett. 82, 398 (1999).

[16] J. Fransson, Int. J. Quant. Chem. 92, 471 (2003).

[17] M.L. Polianski, P. Samuelsson, and M. Büttiker, Phys. Rev. B 72, 161302(R) (2005).

[18] M.L. Polianski and M. Büttiker, Phys. Rev. B 76, 205308 (2007).

[19] Our approach is valid for frequencies $\omega_{L / R}$ smaller than the plasma frequency in the leads (typically a few $\mathrm{THz}$ ), but larger than $\Gamma$ (typically a few hundred $\mathrm{MHz}$ ).

[20] K. Flensberg, Phys. Rev. B 68, 205323 (2003).

[21] M. Galperin, M.A. Ratner, and A. Nitzan, J. Phys. Cond. Matt. 19, 103201 (2007).

[22] In principle, the parameters of the "noninteracting" model have to be computed from a self-consistent meanfield treatment of the interactions. We here discuss interaction phenomena beyond such single-particle effects.

[23] Within a circuit model including geometric capacitances $C_{L, R}$ and the gate capacitance $C_{g}$ as interaction parameters, displacement currents lead to the substitution [10] $\Delta_{g}(t) \rightarrow \Delta_{D}(t)=\frac{C_{L} \Delta_{L}(t)+C_{R} \Delta_{R}(t)+C_{g} \Delta_{g}(t)}{C_{L}+C_{R}+C_{g}}$. For high frequencies, $\Delta_{D}(t)$ coincides with the internal dot potential of Refs. 16, 18].

[24] T.A. Costi, J. Phys. C: Solid State Phys. 19, 5665 (1986). 